\title{
American National Standard For Information Systems-
}

\section{Data Format for the Interchange of Fingerprint, Facial \& SMT Information}

$Q C$

100

.456

N0.6011

1997

United States Department of Commerce

Technology Administration

National Institute of Standards and Technology

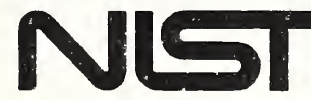





\section{American National Standard For Information Systems-}

\section{Data Format for the Interchange of Fingerprint, Facial \& SMT Information}

National Institute of Standards and Technology

Approved April 7, 1997 American National Standards Institute, Inc.

Issued April 1997

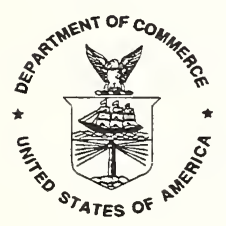

U.S. Department of Commerce

William M. Daley, Secretary

Technology Administration

Mary L. Good, Under Secretary for Technology

National Institute of Standards and Technology

Robert E. Hebner, Acting Director 
This document is a contribution of the National institute of Standards and Technology and is not subject to copyright. Any organization interested in reproducing ANSINIST-ITL 1a-1997, Data Format for the Interchange of Fingerprint, Facial \& SMT Information is free to do so. However, there shall be no alteration to any of the material contained in the document. 


\section{CONTENTS}

FOREWORD

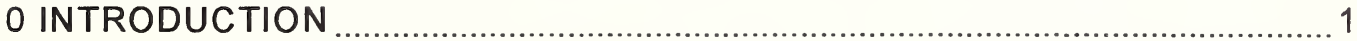

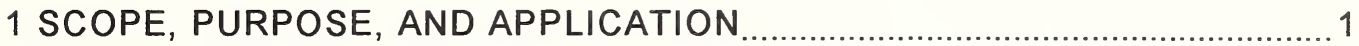

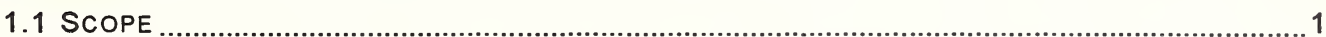

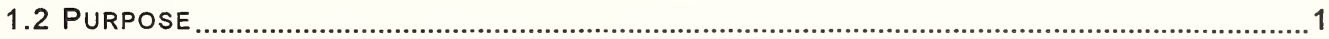

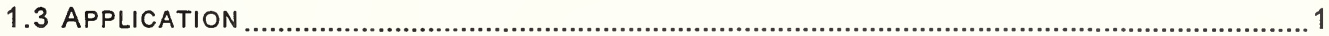

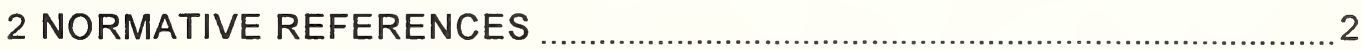

3 DEFINITIONS

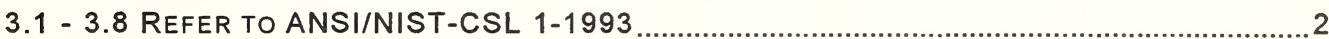

3.9 RGB

3.10 SMT.

3.11 ASPECT RATIO

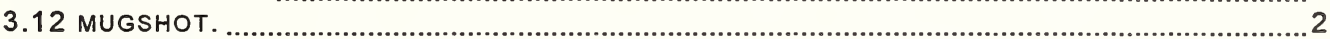

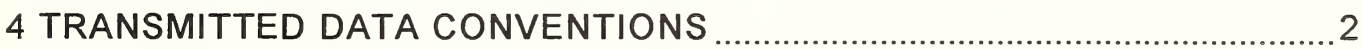

4.1 - 4.3 REFER TO ANSI/NIST-CSL 1-1993 …................................................................ 2

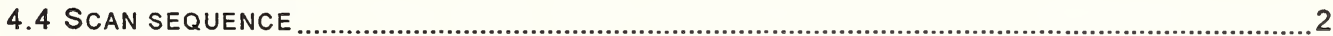

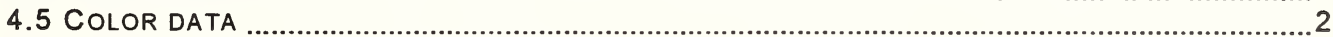

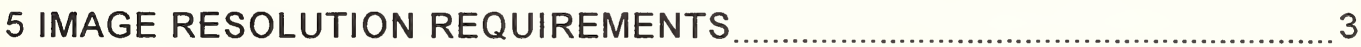

6 FILE DESCRIPTION

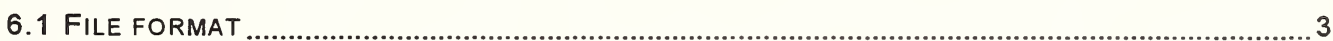

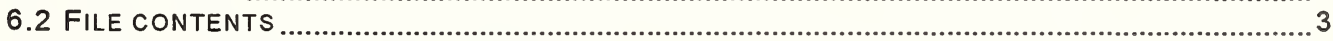

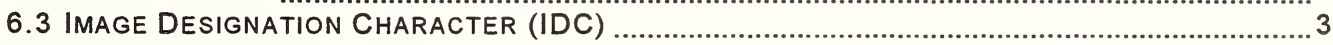

7 RECORD DESCRIPTION

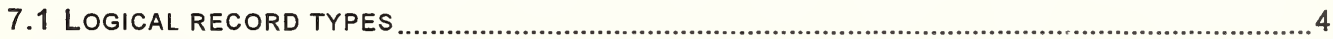

7.2 RECORD FORMAT

8 TYPE-1 TRANSACTION RECORD

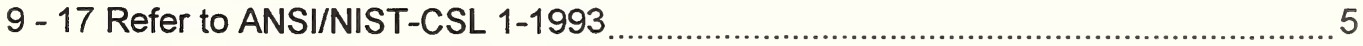

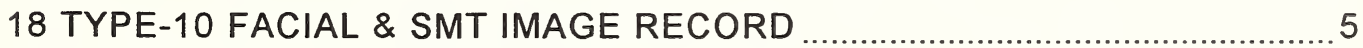

18.1 FIELDS FOR TYPE-10 LOGICAL RECORD ..................................................................... 5

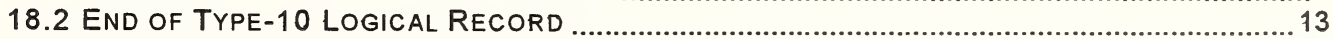

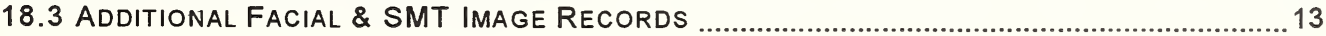

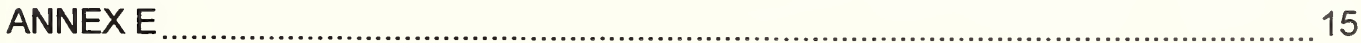

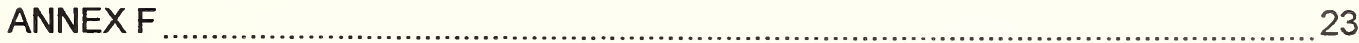




\section{TABLES}

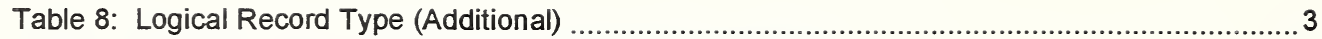

Table 9 Number of Logical Records Per Transaction (Additional) ................................................. 4

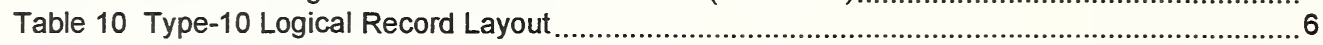

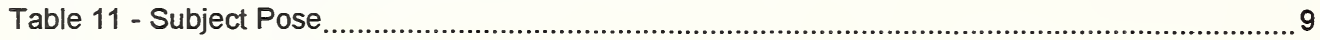

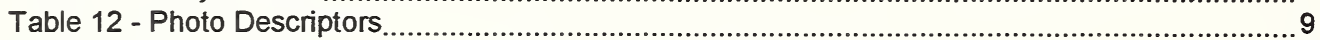

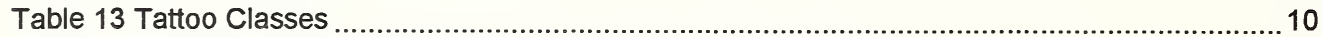

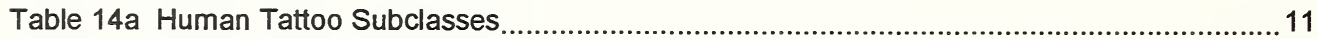

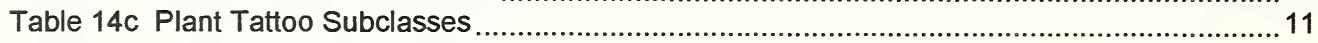

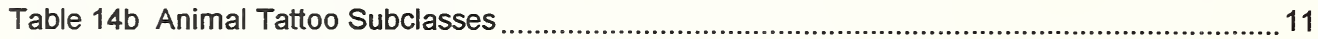

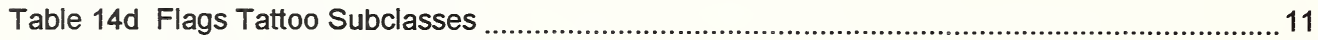

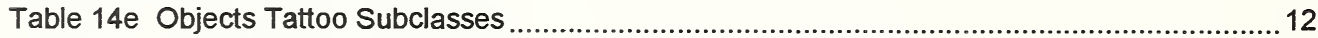

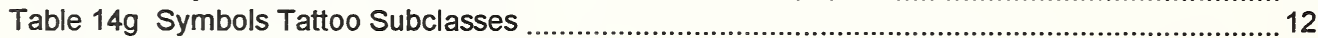

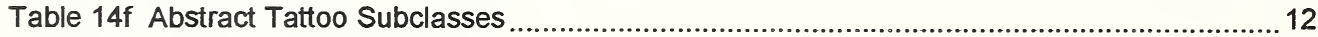

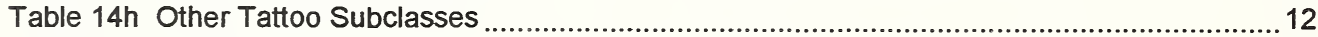

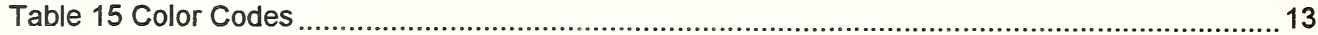


Foreword (This foreword is not part of the American National Standard ANSI/NIST-ITL 1a-1997.)

Federal, state and local law enforcement and related criminal justice agencies have procured or are in the process of procuring electronic image capture, storage, and retrieval systems intended to facilitate the determination of the personal identity of a subject. This equipment will be used to capture a subject's mugshot (or facial image), and any scars, marks, or tattoos (SMT) present on the subject's body.

Digital cameras and other type of video recorders capture images and produce digital image files directly from the subject's head and body. Scanners are used to digitize images from photographs, pictures, or sketches. The digital representations of these image files consist of grayscale or color pixels depending on the application and equipment.

These digital images are stored in a compressed or uncompressed form in an image storage and retrieval system (ISR). Textual descriptive data and other information for each image is also stored. When required, specific images from a master file can be retrieved from the ISR and be incorporated as part of an electronic mugshot book, or an electronic line-up. Images selected may be the result of textual filters based on physical descriptive or information fields indexed to each image. Stored SMT images can also be retrieved as part of an identification process. The actual identification are made by a human witness or an examiner using images retrieved from the system.

To effectively exchange mugshot and SMT identification data across jurisdictional lines or between dissimilar systems produced by different manufacturers, a standard is needed to specify the content and common format for the data exchange. The data may be images captured from digital cameras or the digital representation of scanned photographs or pictures.

The Information Technology Laboratory (ITL), previously the Computer Systems Laboratory, of the National Institute of Standards and Technology (NIST) sponsored the development of the addendum to the Data Format for the Interchange of Fingerprint Information (ANSI/NIST-CSL 1-1993). This addendum enhances the original standard by specifying the data elements required for the exchange of mugshot and SMT information. Consensus during its development was achieved through the use of the Canvass Method.

The sections of this document are numbered in accordance with the corresponding sections in the ANSI/NIST-CSL 1-1993 standard. Section numbers matching those in the standard provide additional information. Section numbers appearing in this addendum, but not in the original standard, provide information that describes the format for the two additional types of image data. Sections appearing in the original standard, but not in this addendum, imply that no additional details are required to accommodate the new image types.

This addendum contains two annexes. Annex E, a description of the Joint Photographic Experts Group (JPEG) File Interchange format, is normative and considered part of the addendum. Annex $F$, contains examples of facial and tattoo images formatted in accordance with this addendum. It is informative and not considered as part of the addendum. 
Suggestions for the improvement of this standard will be welcome. They should be sent to the attention of R.M. McCabe, Mugshot Standards, Visual Image Processing Group, NIST, Building 225, Room A-216, Gaithersburg, MD 20899.

The following organizations recognized as having an interest in the standardization of the data format for the interchange of mugshot and SMT information were contacted prior to the approval of this addendum to the standard. Inclusion in this list does not necessarily imply that the organization concurred with the submittal of the proposed standard to ANSI.

\author{
Abilene Police Department \\ Bureau of Alcohol, Tobacco and Firearms \\ Chicago Police Department \\ Cogent Systems Inc. \\ D.C. Metropolitan Police Department \\ Information Services Division \\ Data Critical Corporation \\ Detroit Police - Identification Unit \\ Digital Biometrics, Inc. \\ Digital Descriptor Systems Inc. \\ Digital Facial Identification System \\ Eastman Kodak Co. \\ ECG Management Consultants \\ Elk Grove Village Police Department \\ EPIC Solutions, Inc. \\ Federal Bureau of Investigation - CJIS \\ Federal Bureau of Investigation - Photography \\ Laboratory \\ Fingermatrix, Inc. \\ Fingerprint USA \\ Florida Department of Law Enforcement \\ Georgia Bureau of Investigation \\ Gregg Thompson Associates \\ Higgins \& Associates, International \\ IDENTIX, Inc. \\ Illinois State Police \\ Indianapolis Police Department \\ Infotec Development, Inc. \\ King County Police \\ LA County Sheriff Department \\ Las Vegas Metropolitan Police \\ Lockheed Martin Energy Systems \\ Manchestor, MO Police Department \\ Massachusetts State Police \\ MD Department of Public Safety \& Correctional \\ Services
}

MD State Police - Crime Laboratory

Metropolitan Police Service, London, England

Michigan State Police - AFIS

Missouri State Highway Patrol

Mitretek Systems, Inc.

National Institute of Justice

North American Morpho Systems, Inc.

NY State Division Criminal Justice Services

Peel Regional Police

Philadelphia Police Department

Positive Identification, Inc.

PRC

PRINTRAK International, Inc.

R.T. Moore Associates

River City Research Group

Riverside County Sheriff

Robert Stock Associates

Royal Canadian Mounted Police

San Antonio, TX Police Department

Saber Imaging

San Jose State University

Sayers Advanced Systems

Search

Sirchie Finger Print Labs

State of Connecticut Department of Public Safety

Tacoma, WA Police Department

Texas Department of Public Safety

TFP Inc.

TRW SIG

UNISYS Corp.

Virginia State Police

Visible Edge, Inc.

Vision Control Int. Pty. Lt.

VT Department of Public Safety

Western Identification Network (WIN)

XImage Corporation 


\section{Data Format for the Interchange of Fingerprint, Facial \& SMT Information (ANSI/NIST-ITL 1a-1997)}

\section{Introduction}

In 1993, ANSI approval was obtained for the "Data Format for the Interchange of Fingerprint Information" standard (ANSI/NIST-CSL 1-1993). The standard specifies formats to be used for exchanging fingerprint and signature image data. Provisions are also included for the exchange of textual or ASCII data describing a subject's demographic characteristics and a limited amount of minutiae or fingerprint characteristic data. The data to be exchanged are contained in nine defined record types and formatted according to specific requirements.

As a result of agreements reached at the Mugshot and Facial Image Workshop held in Gaithersburg on October 23-25, 1995 , the standard has been expanded to address two additional types of biometric image data. This expansion of the standard is in the form of an addendum that specifies a record structure capable of handling the exchange of both types of images.

The first type consists of facial image or mugshot data. The second type pertains to scar, mark, and tattoo (SMT) image data. A provision has also been introduced to enable the exchange of non-specific, user-definable type images that do not fall under any of the defined image type.

\section{application}

\subsection{Scope}

This addendum defines the content, format, and units of measurement for the exchange of information that may be used in the identification of a subject based on facial/mugshot and/or SMT image information. This data shall consist of a variety of mandatory and optional items, including related descriptive data, scanning parameters, and compressed or uncompressed digitized images. This information is intended for interchange between criminal justice administrations or organizations that use facial/mugshot or SMT data for identification purposes.

\subsection{Purpose}

Information compiled and formatted in accordance with this addendum can be recorded on machine-readable media and may be transmitted over data communications facilities. Law enforcement and criminal justice agencies may use it to exchange facial/mugshot and SMT images and related identification data.

\subsection{Application}

Systems claiming conformance with this addendum shall be capable of 
transmitting and receiving Type-1, and Type-10 records and shall specify which other record types are implemented for transmitting, or receiving, or both. Record types not implemented shall be ignored.

\section{Normative references}

The following standards contain provisions which, through reference in this text, constitute provisions of this American National Standard Addendum.

ANSI/NIST-CSL 1-1993, Information systems - Data Format for the Inter-change of Fingerprint Information ${ }^{1}$

ISO International Standard 10918-1, Information Technology - Digital Compression and Coding of Continuous-Tone Still Images Part 1: Requirements and Guidelines ${ }^{2}$ (Commonly referred to as the Joint Photographic Experts Group (JPEG) algorithm).

\section{Definitions}

The following definitions and those given in ANSI/IAI 2 and ANSI/NIST-CSL 1-1993 apply to this addendum.

\section{1 - 3.8 Refer to ANSI/NIST-CSL 1-1993}

3.9 RGB: Red, Green, Blue. Used to represent color pixels comprised of a specified number of bits to represent each of these primary color components.

${ }^{1}$ Available from the American National Standards Institute, 11 West 42 nd Street, New York, NY 10036.

${ }^{2}$ Available from the American National Standards Institute, 11 West 42 nd Street, New York, NY 10036.
3.10 SMT: Abbreviation used for scar, mark, and tattoo information.

3.11 aspect ratio: The width to height ratio of the captured image.

3.12 mugshot: Term used interchangeably with facial image. The term facial image usually implies a higher quality image than a mugshot.

\section{Transmitted data conventions}

\section{1 - 4.3 Refer to ANSI/NIST-CSL 1-1993}

\subsection{Scan sequence}

For each grayscale or color facial or each grayscale or color SMT image that was captured and formatted, the transmitted scan sequence shall be assumed to have been left to right and top to bottom.

For the purpose of describing the position of each pixel within an image, a pair of reference axes shall be used. The origin of the axes, pixel location $(0,0)$, shall be located at the upper left-hand corner of each image. The $x$-coordinate (horizontal) position shall increase positively from the origin to the right side of the image. The $y$-coordinate (vertical) position shall increase positively from the origin to the bottom of the image.

\subsection{Color data}

For color facial or SMT images scanned, it shall be assumed that the scanned images consist of nominal 24-bit RGB 
pixels. For each component of the RGB pixel, its value shall be quantized to eight bits.

Color image data may be transmitted in either compressed or uncompressed form. The transmission of uncompressed color images shall consist of RGB pixels, each of which shall be quantized to 256 levels ( 8 bits) for each of the three components. For each pixel, the three components shall be sequentially formatted for transmission on a pixel-bypixel basis. Compressed image data shall adhere to the requirements of the algorithm used.

\section{Image resolution requirements}

For facial and SMT images, the scanner and transmitting resolution requirements are not applicable.

\section{$6 \quad$ File description}

This addendum describes one additional logical record. It was designed to exchange a combination of ASCII and binary image data within a single logical record structure. At the beginning of the record, a series of tagged fields shall be used to provide information required to process the binary image data present in the last field of the logical record. This logical record type with its identifier is listed in Table 8, Logical Record Type (Additional). This table is an extension of Table 1 in the original standard.

\subsection{File format}

The data fields in the Type-10 record shall be recorded in variable-length fields using the 7-bit American National Standard Code for Information Interchange (ASCII) as described in ANSI
X3.4 and annex A. ${ }^{3}$ For each facial and SMT image record that is formulated, the fields contained within that record shall be numerically ordered and their contents shall be as specified in this addendum.

\section{Table 8: Logical Record Type (Additional)}

\begin{tabular}{|c|c|}
\hline $\begin{array}{c}\text { Logical record } \\
\text { contents }\end{array}$ & Type identifier \\
\hline $\begin{array}{c}\text { Facial \& SMT Image } \\
\text { Data }\end{array}$ & 10 \\
\hline
\end{tabular}

This record type shall contain data fields encoded as ASCII textual information. The concluding field of the Type-10 logical record shall have a tagged ASCII identifier followed by the binary image data.

\subsection{File contents}

Table 9, Number of logical records per transaction (Additional), is an extension of Table 2 of the 1993 standard. It lists the number of Type-10 facial and SMT image records that may occur in a transaction file. The appearance of "O-N" in Table 9 indicates that there is no limit on the number of Type-10 logical records that may be contained in the logical file.

\subsection{Image Designation Character (IDC)}

The IDC shall also be used to relate information items in the file contents field of the Type-1 record to each facial or SMT image record. It properly identifies and links together different logical record types created from the same image. As the facial or SMT image can only be represented by the Type-10 logical record, each such image shall have a

\footnotetext{
${ }^{3}$ This annex can be found in the original standard ANSI/NIST-CSL 1-1993.
} 
Table 9 Number of Logical Records Per Transaction (Additional)

\begin{tabular}{|c|c|c|c|c|c|c|}
\hline $\begin{array}{l}\text { Type of } \\
\text { Logical } \\
\text { Record }\end{array}$ & $\begin{array}{l}\text { Ten-Print } \\
\text { Inquiry }\end{array}$ & $\begin{array}{l}\text { Latent } \\
\text { Inquiry }\end{array}$ & $\begin{array}{l}\text { File } \\
\text { Maintenance }\end{array}$ & $\begin{array}{l}\text { Image } \\
\text { Request }\end{array}$ & $\begin{array}{l}\text { Search } \\
\text { Response }\end{array}$ & $\begin{array}{l}\text { Image } \\
\text { Request } \\
\text { Response }\end{array}$ \\
\hline 10 & $0-\mathrm{N}$ & $0-\mathrm{N}$ & $0-\mathrm{N}$ & $0-\mathrm{N}$ & $0-\mathrm{N}$ & $0-\mathrm{N}$ \\
\hline
\end{tabular}

unique IDC code. The value of the IDC shall be one greater than the last IDC used for the Type-2 through Type- 9 logical records in the file. Subsequent IDCs shall be sequentially incremented by one for each new facial or SMT image.

\section{$7 \quad$ Record description}

\subsection{Logical record types}

\subsection{1 - 7.1.9 Refer to ANSI/NIST-CSL 1993}

\subsubsection{Type-10 facial and SMT image record}

Type-10 logical records shall contain, and be used to exchange facial and SMT image data together with textual information pertinent to the digitized image. The source of the image data shall be the image captured from scanning a photograph, or a live image captured with a digital camera.

\subsection{Record format}

\subsubsection{Refer to ANSI/NIST-CSL 1-1993}

\subsubsection{Record layout}

The Type-10 record shall use a combination of tagged ASCII field identifiers (as used in the Type-1, Type2 , and Type-9 logical records) followed by ASCII descriptive data for each field. The record shall end with a tagged ASCII field identifier preceding the binary image data. Each Type-10 record information field that is used, shall be numbered in accordance with this standard. The format of each field shall consist of a field number followed by a colon (:), followed by the information item(s) appropriate to that field. For this logical record, field numbers have the format "10.XXX:", where 10 is the logical record type, and "XXX" is a sequentially assigned field number within that record type. Multiple occurrences of the same subfield within a logical record shall be separated by the $R S$ character. Individual information items within a field or subfield shall be separated by the US character. In addition to the identifying number, information fields shall be separated from other information fields by the ASCII Group Separator (GS) control character.

For each field or subfield that is designated as mandatory, the appropriate information shall be entered in the required format. An optional field shall be omitted when the information for that field is unavailable or unneeded. Each logical Type-10 record shall contain a single image. The last field in the logical record (field 10.999:) shall contain the image data which shall be placed immediately following the colon (":") of the field identifier. The length of the entire record shall be specified by the record length field contained in the record itself. The ASCII File Separator (FS) control character shall follow the last byte of the compressed or uncompressed image data. The FS character shall signify the end of the logical record. 


\section{Type-1 Transaction record}

\subsubsection{Refer to ANSI/NIST-CSL 1-1993}

\subsubsection{Field 1.02: Version Number (VER)}

The entry in this field shall be "0201" to indicate the current version of the standard used to encode the image data. This version number signifies the inclusion of the Type-10 logical record.

\subsubsection{Field 1.03: File Content (CNT)}

This mandatory field shall list each of the logical records in the logical file by record type. The count of the logical records, which is contained in the second information item of the first subfield, shall include the number of Type-10 records contained in the logical file. A subfield shall be included in Field 1.03 for each Type-10 record present in the logical file. The first information item shall be the two-character code "10" chosen from Table 8 , which declares the record type. The second information item shall be the IDC associated with the logical record pertaining to that subfield. The US character shall be used to separate the two information items.

\subsection{4 - 8.1.10 Refer to ANSI/NIST- CSL 1-1993}

\subsubsection{Field 1.11: Native Scanning Resolution (NSR)}

This field applies only to fingerprint image data. For those logical files that contain only Type-10 image records, this field shall be set to "00.00".

\subsubsection{Field 1.12: Nominal Transmitting Resolution (NTR)}

This field applies only to fingerprint image data. For those logical files that contain only Type-10 image records, this field shall be set to "00.00".

\section{9-17 Refer to ANSI/NIST-CSL 1- 1993}

\section{Type-10 facial \& SMT image record}

Type-10 records shall contain facial and/or SMT binary image data and related $A S C I I$ information pertaining to the specific image contained in this record. It shall be used to exchange both grayscale and color image data. Image data contained in the Type- 10 record may be uncompressed or compressed.

\subsection{Fields for Type-10 logical record}

When there are one or more Type-10 logical records, entries shall be provided in ordered numbered fields. For each field of the Type- 10 record, Table 10 summarizes the condition code as being mandatory $(M)$ or optional $(O)$; the field number, the field name, character type, size and occurrence limits, and the maximum size in bytes of the field. The two entries in the Field Size Per Occurrence include all character separators used in the field. The Maximum Byte Count includes the field number, the information, and all the character separators. Fields containing entries in the IMG column are only applicable to that image type. An entry of "FAC" applies to a mugshot or facial image, and an entry of "SMT" applies to scar, a mark, or a tattoo image. The following paragraphs describe the data 
Table 10 Type-10 Logical Record Layout

\begin{tabular}{|c|c|c|c|c|c|c|c|c|c|c|}
\hline \multirow[t]{2}{*}{ IDENT } & \multirow[t]{2}{*}{$\begin{array}{l}\text { COND } \\
\text { CODE }\end{array}$} & \multirow[t]{2}{*}{$\begin{array}{l}\text { FIELD } \\
\text { NUMBER }\end{array}$} & \multirow[t]{2}{*}{ FIELD NAME } & \multirow[t]{2}{*}{ IMG } & \multirow[t]{2}{*}{$\begin{array}{l}\text { CHAR } \\
\text { TYPE }\end{array}$} & \multicolumn{2}{|c|}{$\begin{array}{l}\text { FIELD SIZE PER } \\
\text { OCCURRENCE }\end{array}$} & \multicolumn{2}{|c|}{$\begin{array}{l}\text { OCCUR } \\
\text { COUNT }\end{array}$} & \multirow[t]{2}{*}{$\begin{array}{l}\text { MAX BYTE } \\
\text { COUNT }\end{array}$} \\
\hline & & & & & & lain. & $\operatorname{MAX}$ & MIN & $\max$ & \\
\hline LEN & M & 10.001 & LOGICAL RECORD LENGTH & & $\mathrm{N}$ & 4 & 8 & 1 & 1 & 15 \\
\hline IDC & M & 10.002 & $\begin{array}{l}\text { IMAGE DESIGNATION } \\
\text { CHARACTER }\end{array}$ & & $\mathrm{N}$ & 2 & 5 & 1 & 1 & 12 \\
\hline IMT & M & 10.003 & IMAGE TYPE & & A & 5 & 7 & 1 & 1 & 14 \\
\hline SRC & M & 10.004 & SOURCE AGENCY / ORI & & AN & 10 & 21 & 1 & 1 & 28 \\
\hline PHD & M & 10.005 & PHOTO DATE & & $\mathrm{N}$ & 9 & 9 & 1 & 1 & 16 \\
\hline HLL & $M$ & 10.006 & HORIZONTAL LINE LENGTH & & $\mathrm{N}$ & 4 & 5 & 1 & 1 & 12 \\
\hline VLL & $M$ & 10.007 & VERTICAL LINE LENGTH & & $\mathrm{N}$ & 4 & 5 & 1 & 1 & 12 \\
\hline SLC & $M$ & 10.008 & SCALE UNITS & & $\mathrm{N}$ & 2 & 2 & 1 & 1 & 9 \\
\hline HPS & M & 10.009 & HORIZONTAL PIXEL SCALE & & $N$ & 3 & 5 & 1 & 1 & 12 \\
\hline VPS & M & 10.010 & VERTICAL PIXEL SCALE & & $\mathrm{N}$ & 3 & 5 & 1 & 1 & 12 \\
\hline CGA & $M$ & 10.011 & COMPRESSION ALGORITHM & & A & 5 & 7 & 1 & 1 & 14 \\
\hline $\operatorname{csp}$ & M & 10.012 & COLOR SPACE & & A & 4 & 5 & 1 & 1 & 12 \\
\hline RSV & - & $\begin{array}{l}10.013 \\
10.019\end{array}$ & $\begin{array}{l}\text { RESERVED FOR FUTURE } \\
\text { INCLUSION }\end{array}$ & & -- & -- & -- & -- & -- & -- \\
\hline POS & 0 & 10.020 & SUBJECT POSE & FAC & A & 2 & 2 & 0 & 1 & 9 \\
\hline POA & 0 & 10.021 & POSE OFFSET ANGLE & FAC & $\mathrm{N}$ & 2 & 5 & 0 & 1 & 12 \\
\hline PXS & O & 10.022 & PHOTO DESCRIPTION & FAC & A & 4 & 21 & 0 & 9 & 196 \\
\hline RSV & - & $\begin{array}{l}10.023 \\
10.039\end{array}$ & $\begin{array}{l}\text { RESERVED FOR FUTURE } \\
\text { INCLUSION }\end{array}$ & & -- & -- & -- & -- & -- & --- \\
\hline SMT & M & 10.040 & NCIC DESIGNATION CODE & SMT & A & 4 & 11 & 1 & 3 & 40 \\
\hline SMS & 0 & 10.041 & SCAR/MARKITATTOO SIZE & SMT & $\mathrm{N}$ & 4 & 6 & 0 & 1 & 13 \\
\hline SMD & 0 & 10.042 & SMT DESCRIPTORS & SMT & AN & 16 & 51 & 0 & 9 & 466 \\
\hline COL & 0 & 10.043 & COLORS PRESENT & SMT & A & 4 & 21 & 0 & 9 & 196 \\
\hline RSV & - & $\begin{array}{l}10.044 \\
10.199\end{array}$ & $\begin{array}{l}\text { RESERVED FOR FUTURE } \\
\text { INCLUSION }\end{array}$ & & -- & -- & -- & -- & -- & --- \\
\hline UDF & 0 & $\begin{array}{l}10.200 \\
10.998\end{array}$ & USER DEFINED FIELDS & & -- & - & $\cdots$ & -- & -- & $\cdots$ \\
\hline DAT & $M$ & 10.999 & IMAGE DATA & & B & 2 & $5,000,001$ & 1 & 1 & $5,000,008$ \\
\hline
\end{tabular}

KEY FOR CHARACTER TYPE: N=NUMERIC; $A=A L P H A B E T I C ; A N=A L P H A N U M E R I C ; B=B I N A R Y$ 
contained in each of the fields for the Type-10 logical record. Each field shall begin with a seven character ASCII identifier of the form "10.xxx:". The first two characters are the record type followed by a period. The next three characters are the appropriate field number followed by a colon. Descriptive ASCII information or the binary image data follows the seven character identifier.

\subsubsection{Field 10.001: Logical Record Length (LEN)}

This mandatory ASCII field shall contain the total count of the number of bytes in the Type-10 logical record. Field 10.001 shall specify the length of the record including every character of every field contained in the record and the information separators. The GS character shall separate the length code of Field 10.001 from the next field.

\subsubsection{Field 10.002: Image Designation Character (IDC)}

This mandatory one to four byte ASCII field shall be used to identify the facial or SMT image data contained in the record. This IDC shall match the IDC found in the file content field of the Type-1 record.

\subsubsection{Field 10.003: Image Type (IMT)}

This mandatory ASCII field is used to indicate the type of image contained in this record. It shall contain "FACE", "SCAR", "MARK", or "TATTOO" to indicate a face, scar, mark or tattoo image.

The field may also contain "OTHER" to indicate a user-defined miscellaneous type image. This entry provides the ability to use the Type-10 record structure to exchange images not addressed by other record types in the standard. However, additional field numbers for ASC11 descriptors must be greater than 200. The content of these fields shall conform to the requirements set forth by the agency to whom the transmission is being sent.

\subsubsection{Field 10.004: Source Agency / ORI (SRC)}

This mandatory ASCII field shall contain the identification of the administration or organization that originally captured the facial image contained in the record. Normally, the Originating Agency Identifier (ORI) of the agency that captured the image will be contained in this field. The size and data content of this field shall be defined by the user and be in accordance with the receiving agency.

\subsubsection{Field 10.005: Photo Date (PHD)}

This mandatory ASCII field shall contain the date that the facial or SMT image contained in the record was captured. The date shall appear as eight digits in the format CCYYMMDD. The CCYY characters shall represent the year the image was captured; the $M M$ characters shall be the tens and units values of the month; and the $D D$ characters shall be the tens and units values of the day in the month. For example, 19960229 represents February 29, 1996. The complete date must be a legitimate date and shall not exceed the current date.

\subsubsection{Field 10.006: Horizontal Line Length (HLL)}

This mandatory ASCII field shall contain the number of pixels contained on a single horizontal line of the transmitted image.

\subsubsection{Field 10.007: Vertical Line Length (VLL)}

This mandatory ASCII field shall contain the number of horizontal lines contained in the transmitted image. 


\subsubsection{Field 10.008: Scale Units (SLC)}

This mandatory ASCII field shall specify the units used to describe the image sampling frequency (pixel density). A " 1 " in this field indicates pixels per inch, or a "2" indicates pixels per centimeter. A " 0 " in this field indicates no scale is given. For this case, the quotient of HPS/VPS gives the pixel aspect ratio.

\subsubsection{Field 10.009: Horizontal Pixel Scale (HPS)}

This mandatory ASCII field shall specify the pixel density used in the horizontal direction providing the SLC contains a "1" or a "2". Otherwise, it indicates the horizontal component of the pixel aspect ratio.

\subsubsection{Field 10.010: Vertical Pixel Scale (VPS)}

This mandatory ASCII field shall specify the pixel density used in the vertical direction providing the SLC contains a "1" or a "2". Otherwise, it indicates the vertical component of the pixel aspect ratio.

\subsubsection{Field 10.011: Compression Algorithm (CGA)}

This mandatory ASCII field shall specify the algorithm used to compress the color or grayscale image. An entry of "NONE" in this field indicates that the data contained in this record is uncompressed. For those images that are to be compressed, the preferred method for the compression of facial and SMT images is specified by the baseline mode of the JPEG algorithm formatted in accordance with the JPEG File Interchange Format, Version 1.02 (JFIF). ${ }^{4}$ An entry of "JPEGB" indicates that the scanned or

\footnotetext{
${ }^{4}$ Developed by C-Cube Microsystems, 1778 McCarthy Blvd., Milpitas, CA 95035.
}

captured image was compressed using baseline JPEG. An entry of "JPEGL" indicates that the lossless mode of the JPEG algorithm was used to compress the image. If the image is captured in grayscale, then only the luminescence component will be compressed and transmitted. The FBI will maintain a registry of additional compression techniques and corresponding codes that may be used as they become available.

\subsubsection{Field 10.012: Colorspace (CSP)}

This mandatory ASCII field shall contain the color space used to exchange the image. For compressed images, the preferred colorspace using baseline JPEG and JFIF is $Y C b C r^{5}$ to be coded as "YCC". An entry of "GRAY" shall be used for all grayscale images. This field shall contain "RGB" for uncompressed color images containing non-interleaved red, green, and blue pixels in that order. All other colorspaces are undefined.

\subsubsection{Field 10.013-.019: Reserved for Future Definition (RSV)}

These fields are reserved for inclusion in future revisions of this standard. None of these fields are to be used at this revision level. If any of these fields are present, they are to be ignored.

\subsubsection{Field 10.020: Subject Pose (POS)}

This optional field is to be used for the exchange of facial image data. When included, this field shall contain a one ASCII character code selected from Table-11 to describe the pose of the subject. For the angled pose entry "A", field 10.021 shall contain the offset angle from the full face orientation.

\footnotetext{
${ }^{5}$ Annex $F$ contains the information necessary to perform conversions between 24-bit RGB pixels and the $\mathrm{YCbCr}$ color space.
} 
Table 11 - Subject Pose

\begin{tabular}{|l|c|}
\hline \multicolumn{1}{|c|}{ POSE DESCRIPTION } & POSE CODE \\
\hline \hline Full Face Frontal & $\mathrm{F}$ \\
\hline Right Profile (90 degree) & $\mathrm{R}$ \\
\hline Left Profile (90 degree) & $\mathrm{L}$ \\
\hline Angled Pose & $\mathrm{A}$ \\
\hline
\end{tabular}

\subsubsection{Field 10.021: Pose Offset Angle (POA)}

This field shall only be used for the exchange of facial image data if Field 10.020 (POS) contains an " $A$ " to indicate an angled pose of the subject. This field should be omitted for a full face or a profile. This ASCII field specifies the pose position of the subject at any possible orientation within a circle. Its value shall be to a nearest degree.

The offset angle shall be measured from the full-face pose position and have a range of values from -180 degrees to +180 degrees. A positive angle is used to express the angular offset as the subject rotates from a full-face pose to their right (approaching a left profile). A negative angle is used to express the angular offset as the subject rotates from a full-face pose to their left (approaching a right profile). If the entry in the POS field is an " $F$ ", " $L$ ", or " $R$ ", the contents of this field are ignored.

\subsubsection{Field 10.022: Photo Description (PXS)}

This optional ASCII field shall be used for the exchange of facial image data. When present, it shall describe special attributes of the captured facial image. Attributes associated with the facial image may be selected from Table 12 and entered in this field.
Table 12 - Photo Descriptors

\begin{tabular}{||l|l|}
\hline FACIAL IMAGE ATTRIBUTE & ATTRIBUTE CODE \\
\hline \hline Subject Wearing Glasses & GLASSES \\
\hline Subject Wearing Hat & HAT \\
\hline Subject Wearing Scarf & SCARF \\
\hline Physical Characteristics & PHYSICAL \\
\hline Other Characteristics & OTHER \\
\hline
\end{tabular}

Physical characteristics, such as "FRECKLES" may be entered as a subfield consisting of two information items. The first is "PHYSICAL" followed by the US separator, followed by the characteristic as listed in Part 4 Section 13 of the NCIC Code Manual (Third Edition; July 1984). The "OTHER" category is used to enter unlisted or miscellaneous attributes of the facial image. This information shall be entered as a two information item subfield. The first is "OTHER" followed by the US separator, followed by the unformatted text used to describe the attribute. Multiple attributes and subfields may be listed but must be separated by the $R S$ character.

\subsubsection{Field 10.023-.039: Reserved for Future Definition (RSV)}

These fields are reserved for inclusion in future revisions of this standard. None of these fields are to be used at this revision level. If any of these fields are present, they are to be ignored.

\subsubsection{Field 10.040: NCIC Designation Code (SMT)}

This field is mandatory for a Type -10 record containing SMT image data. It is used to identify a general location of the captured scar, mark, or tattoo image. The contents of this field will be an entry chosen from Part 4 Section 13 of the NCIC Code Manual (Third Edition; July 1984). The captured image can encompass an area larger than that 
specified by a single NCIC body part code for the particular image type. This situation can be accommodated by listing multiple NCIC codes separated by the RS separator character. In this case the primary code is listed first.

For the "marks" category, the NCIC manual lists the common locations for needle track marks. The body location codes listed for scars shall be used for other body part locations or other types of marks not listed in the NCIC Code Manual.

\subsubsection{Field 10.041: SMT Size (SMS)}

This optional field shall contain the dimensions of the scar, mark or tattoo. It shall consists of two information items.

The height shall be the first information item followed by the US separator character followed by the width. Each dimension shall be entered to the nearest centimeter.

\subsubsection{Field 10.042: SMT Descriptors (SMD)}

This optional field is used to describe the SMT image. It shall consists of one or more subfields. Each subfield shall contain three or four information items that provide progressively detailed information describing the total image or a portion of the image.

The first information item of each subfield shall identify the source of the SMT. This item will contain "TATTOO" for the creation of a common tattoo or indelible image resulting from the pricking of the skin with a coloring matter; "CHEMICAL" if the SMT was created by the use of chemicals to burn the image into the skin; or "BRANDED" if the image was burned into the skin using a branding iron or other form of heat. The second information item shall be the general class code of tattoo chosen from Table 13. For each general class of tattoo, there are several defined subclasses. The third information item of the subfield shall be the appropriate subclass code selected from Tables 14a$14 \mathrm{~h}$ which lists the various subclasses of tattoos for each of the general classes. The final and optional information item in this subfield shall be a free-form ASCII text string that provides additional qualifiers to describe the image or portion of the image. For example, to fully describe a tattoo, there may be a class description of "ANIMAL", with a subclass description of "DOG", and qualified by "golden retriever with an overbite". The information items will be separated by the US separator character.

\section{Table 13 Tattoo Classes}

\begin{tabular}{|c|c|}
\hline $\begin{array}{c}\text { CLASS } \\
\text { DESCRIPTION }\end{array}$ & CLASS CODE \\
\hline $\begin{array}{c}\text { Human Forms and } \\
\text { Features }\end{array}$ & HUMAN \\
\hline $\begin{array}{c}\text { Animals and Animal } \\
\text { Features }\end{array}$ & ANIMAL \\
\hline Plants & PLANT \\
\hline Flags & FLAG \\
\hline Objects & OBJECT \\
\hline Abstractions & ABSTRACT \\
\hline Insignias \& Symbols & SYMBOL \\
\hline Other Images & OTHER \\
\hline
\end{tabular}

An SMT image consisting of several parts or sub-images shall use multiple subfields, separated by the $R S$ separator, to fully describe the various parts or features found in the total image. The first subfield shall describe the most predominant feature or sub-image contained in the SMT. Subsequent subfields shall describe additional portions of the image that are not part of the main or central focal point of the image. For example, a tattoo consisting of a man with a snake on the arm being followed by a dog may contain three subfields - one describing the man, a second describing the snake, and a third describing the dog. 
Table 14a Human Tattoo Subclasses

\begin{tabular}{||l|l||}
\hline \multicolumn{1}{|c|}{ SUBCLASS } & \multicolumn{1}{|c|}{ SUBCLASS CODE } \\
\hline \hline Male Face & MFACE \\
\hline Female Face & FFACE \\
\hline Abstract Face & ABFACE \\
\hline Male Body & MBODY \\
\hline Female Body & FBODY \\
\hline Abstract Body & ABBODY \\
\hline $\begin{array}{l}\text { Roles (Knight, Witch, } \\
\text { man, etc.) }\end{array}$ & ROLES \\
\hline $\begin{array}{l}\text { Sports Figures (Football } \\
\text { Player, Skier, etc.) }\end{array}$ & SPORT \\
\hline Male Body Parts & MBPART \\
\hline Female Body Parts & FBPART \\
\hline Abstract Body Parts & ABBPART \\
\hline $\begin{array}{l}\text { Skulls } \\
\text { Forms }\end{array}$ & SKULL \\
\hline
\end{tabular}

Table 14c Plant Tattoo Subclasses

\begin{tabular}{||l|l||}
\hline \multicolumn{1}{|c|}{ SUBCLASS } & \multicolumn{1}{|c|}{ SUBCLASS CODE } \\
\hline Narcotics & NARCOTICS \\
\hline Red Flowers & REDFL \\
\hline Blue Flowers & BLUEFL \\
\hline Yellow Flowers & YELFL \\
\hline Drawings of Flowers & DRAW \\
\hline Rose & ROSE \\
\hline Tulip & TULIP \\
\hline Lily & LILY \\
\hline $\begin{array}{l}\text { Miscellaneous Plants, } \\
\text { Flowers, Vegetables }\end{array}$ & MPLANT \\
\hline \hline
\end{tabular}

Table 14b Animal Tattoo Subclasses

\begin{tabular}{||l|l|}
\hline \multicolumn{1}{|c|}{ SUBCLASS } & SUBCLASS CODE \\
\hline \hline Cats \& Cat Heads & CAT \\
\hline Dogs \& Dog Heads & DOG \\
\hline Other Domestic Animals & DOMESTIC \\
\hline $\begin{array}{l}\text { Vicious Animals (Lions, } \\
\text { Tigers, Wolves, etc.) }\end{array}$ & VICIOUS \\
\hline $\begin{array}{l}\text { Horses (Donkeys, Mules, } \\
\text { etc.) }\end{array}$ & HORSE \\
\hline Other Wild Animals & WILD \\
\hline Snakes & SNAKE \\
\hline Dragons & DRAGON \\
\hline Birds (Cardinal, Hawk, etc.) & BIRD \\
\hline Spiders, Bugs, and Insects & INSECT \\
\hline Abstract Animals & ABSTRACT \\
\hline Animal Parts & PARTS \\
\hline $\begin{array}{l}\text { Miscellaneous Animal } \\
\text { Forms }\end{array}$ & MANIMAL \\
\hline
\end{tabular}

Table 14d Flags Tattoo Subclasses

\begin{tabular}{||l|l|}
\hline \multicolumn{1}{|c|}{ SUBCLASS } & \multicolumn{1}{|c|}{ SUBCLASS CODE } \\
\hline American Flag & USA \\
\hline State Flag & STATE \\
\hline Nazi Flag & NAZI \\
\hline Confederate Flag & CONFED \\
\hline British Flag & BR!T \\
\hline Miscellaneous Flags & MFLAG \\
\hline
\end{tabular}


Table 14e Objects Tattoo Subclasses

\begin{tabular}{|l|l||}
\hline \multicolumn{1}{|c|}{ SUBCLASS } & \multicolumn{1}{|c|}{$\begin{array}{c}\text { SUBCLASS } \\
\text { CODE }\end{array}$} \\
\hline Fire & FIRE \\
\hline Weapons (Guns, Arrows, etc.) & WEAP \\
\hline Airplanes & PLANE \\
\hline Boats, Ships, and Other Vessels & VESSEL \\
\hline Trains & TRAIN \\
\hline Cars, Trucks, and Vehicles & VEHICLE \\
\hline Mythical (Unicorns, etc.) & MYTH \\
\hline $\begin{array}{l}\text { Sporting Objects (Football, Ski, } \\
\text { Hurdles, etc.) }\end{array}$ & SPORT \\
\hline $\begin{array}{l}\text { Water \& Nature Scenes (Rivers, } \\
\text { Sky, Trees, etc.) }\end{array}$ & NATURE \\
\hline \begin{tabular}{l} 
Miscellaneous Objects \\
\hline
\end{tabular}
\end{tabular}

Table $14 \mathrm{~g}$ Symbols Tattoo Subclasses

\begin{tabular}{|l|l|}
\hline \multicolumn{1}{|c|}{ SUBCLASS } & \multicolumn{1}{|c|}{ SUBCLASS CODE } \\
\hline National Symbols & NATION \\
\hline Political Symbols & POLITIC \\
\hline Military Symbols & MILITARY \\
\hline Fraternal Symbols & FRATERNAL \\
\hline Professional Symbols & PROFESS \\
\hline Gang Symbols & GANG \\
\hline Miscellaneous Symbols & MSYMBOLS \\
\hline
\end{tabular}

\subsubsection{Field 10.043: Color (COL)}

This optional field shall contain one subfield corresponding to each subfield contained in Field 10.042. Each subfield shall contain one or more information items that list the color(s) of the tattoo or part of the tattoo. For each subfield, the first information item in the subfield shall
Table 14f Abstract Tattoo Subclasses

\begin{tabular}{||l||l||}
\hline \multicolumn{1}{|c|}{ SUBCLASS } & \multicolumn{1}{c|}{ SUBCLASS CODE } \\
\hline Figure(s) & FIGURE \\
\hline Sleeve & SLEEVE \\
\hline Bracelet & BRACE \\
\hline Anklet & ANKLET \\
\hline Necklace & NECKLC \\
\hline Shirt & SHIRT \\
\hline Body Band & BODBND \\
\hline Head Band & HEDBND \\
\hline Miscellaneous Abstract & MABSTRACT \\
\hline
\end{tabular}

Table 14h Other Tattoo Subclasses

\begin{tabular}{|l|l|}
\hline \multicolumn{1}{|c|}{ SUBCLASS } & \multicolumn{1}{|c|}{ SUBCLASS CODE } \\
\hline \hline $\begin{array}{l}\text { Wording (Mom, Dad, } \\
\text { Mary, etc.) }\end{array}$ & WORDING \\
\hline Freeform Drawings & FREEFRM \\
\hline Miscellaneous Images & MISC \\
\hline
\end{tabular}

be the predominant color chosen from Table 15. Additional colors for the subfield shall be entered as information items in the subfield separated by the US separator character.

\subsubsection{Field 10.044-199: Reserved for Future Definition (RSV)}

These fields are reserved for inclusion in future revisions of this standard. None of these fields are to be used at this revision level. If any of these fields are present, they are to be ignored. 
Table 15 Color Codes

\begin{tabular}{|l|l|}
\hline $\begin{array}{l}\text { Color } \\
\text { Description }\end{array}$ & Color Code \\
\hline \hline Black & BLACK \\
\hline Brown & BROWN \\
\hline Gray & GRAY \\
\hline Blue & BLUE \\
\hline Green & GREEN \\
\hline Orange & ORANGE \\
\hline Purple & PURPLE \\
\hline Red & RED \\
\hline Yellow & YELLOW \\
\hline White & WHITE \\
\hline Multi-colored & MULTI \\
\hline Outlined & OUTLINE \\
\hline
\end{tabular}

\subsubsection{Field 10.200-998: User Defined Fields (UDF)}

These fields are user-definable fields. Their size and content shall be defined by the user and be in accordance with the receiving agency. If present they shall contain ASCII textual information.

\subsubsection{Field 10.999: Image Data (DAT)}

This field shall contain all of the grayscale or color data from a face, scar, mark, tattoo, or other image. It shall begin with the ASCII identifier "10.999:", and be followed by image data in a binary representation.
Each pixel of uncompressed grayscale data shall be quantized to eight bits (256 gray levels) contained in a single byte.

Uncompressed color image data shall be expressed as 24 bit RGB pixels. The first byte shall contain the eight bits for the red component of the pixel, the second byte shall contain the eight bits for the green component of the pixel, and the third byte shall contain the last eight bits for the blue component of the pixel. If compression is used, the pixel data shall be compressed in accordance with the compression technique specified in the GCA field. If the JPEG algorithm is be used to compress the data, this field shall be encoded using the JFIF format specification.

\subsection{End of Type-10 Logical Record}

For the sake of consistency, immediately following the last byte of data from field 10.999 an FS separator shall be used to separate it from the next logical record. This separator must be included in the length field of the Type-10 record.

\subsection{Additional Facial \& SMT Image Records}

Additional Type-10 records may be included in the logical file. For each additional facial image, a complete Type-10 logical record together with the FS separator is required. 
ANSI/NIST-ITL 1a-1997 


\section{Annex E}

JPEG File Interchange Format

Version 1.02

September 1, 1992

Eric Hamilton

C-Cube Microsystems

1778 McCarthy Blvd.

Milpitas, CA 95035

$+1408944-6300$

Fax: +1408 944-6314

E-mail: eric@c3.pla.ca.us 


\title{
JPEG File Interchange Format
}

\author{
Version 1.02
}

\section{Why a File Interchange Format}

JPEG File Interchange Format (JFIF) is a minimal file format which enables JPEG bitstreams to be exchanged between a wide variety of platforms and applications. This minimal format does not include any of the advanced features found in the TIFF JPEG specification or any application specific file format. The only purpose of this simplified format is to allow the exchange of JPEG compressed images.

\section{JPEG File Interchange Format features}

- Uses JPEG compression

- Uses JPBG interchange format compressed image representation

- PC or Mac or UNIX workstation compatible

- Standard color space: one or three components. For three components YCbCr (CCIR 601-256 levels)

- APPO marker used to specify Units, $X$ pixel density, $Y$ pixel density, thumbnail

- APPO marker also used to specify JFIF extensions

- APPO mater also used to specify application-specific information

\section{JPEG Compression}

Although any JPEG process is supported by the syntax of the JFIF it is strongly recommended that the JPEG baseline process be used for the purposes of file interchange. This ensures maximum compatibility with all applications supporting JPEG. JFIF conforms to the JPEG Draft International Standard (ISO DIS 10918-1).

The JFIF is entirely compatible with the standard JPEG interchange format; the only additional requirement is the mandatory presence of the APPO marker right after the SOI marker. Note that the JPEG interchange format requires (as does JFIF) all table specifications used in the encoding process be coded in the bitstream prior to their use.

\section{Compatible across Platforms}

The JFIF is compatible across platforms: for example, it can use any resource forks supported by the Macintosh and by PCs or workstations, but not just one platform.

\section{Standard color Space}

The color space to be used is $\mathrm{YCbCr}$ as defined by CCIR 601 (256 levels). The RGB components calculated by linear conversion from $\mathrm{YCbCr}$ shall not be gamma corrected (gamma =1.0). If only one component is used, that component shall be $Y$. 
APPO marker is used to identify JPEG FIF

The APPO marker is used to identify a JPEG FIF file.

The JPEG FIF APPO marker is mandatory right after the SOI marker.

The JFIF APPO marker is identified by a zero terminated string: "JFIF".

The APPO can be used for any other purpose by the application provided it can be distinguished from the JFIF APPO.

The JFIF APPO marker provides information which is missing from the JPEG stream: version number, $X$ and $Y$ pixel density (dots per inch or dots per $\mathrm{cm}$ ), pixel aspect ratio (derived from $X$ and $Y$ pixel density), thumbnail.

\section{APPO marker used to specify JFIF extensions}

Additional APPO marker segment(s) can optionally be used to specify JFIF extensions. If used, these segments must immediately follow the JFIF APPO marker. Decoders should skip any unsupported JFIF extension segments and continue decoding.

The JFIF extension APPO marker is identified by a zero terminated string: "JFXX". The JFIF extension APPO marker segment contains a 1-byte code which identifies the extension. This version, version 1.02 , has only one extension defined: an extension for defining thumbnails stored in formats other than 24-bit RGB.

\section{APPO marker used for application-specific information}

Additional APPO marker segments can be used to hold application-specific information which does not affect the decodability or displayability of the JFIF file. Applicationspecific APPO marker segments must appear after the JFIF APPO and any JFXX APPO segments. Decoders should skip any unrecognized application-specific APPO segments.

Application-specific APPO marker segments are identified by a zero terminated string which identifies the application (not "JFIF" or "JFXX"). This string should be an organization name or company trademark. Generic strings such as dog, cat, tree, etc. should not be used.

\section{Conversion to and from RGB}

$\mathrm{Y}, \mathrm{Cb}$, and $\mathrm{Cr}$ are converted from $\mathrm{R}, \mathrm{G}$, and $\mathrm{B}$ as defined in CCIR Recommendation 601 but are normalized so as to occupy the full 256 levels of an 8-bit binary encoding. More precisely:

$$
\begin{aligned}
& Y=256 * E_{y}^{\prime} \\
& C b=256^{*}\left[E^{\prime}{ }_{c b}\right]+128 \\
& C r=256^{*}\left[E^{\prime} c_{r}\right]+128
\end{aligned}
$$

where the $E_{y}^{\prime} E^{\prime} c_{b}$ and $E^{\prime} c_{r}$ are defined as in CCIR 601. Since values of $E_{y}^{\prime}$ have a range 
of 0 to 1.0 and those for $E^{\prime} c_{b}$ and $E_{c r}^{\prime}$ have a range of -0.5 to $+0.5, Y, C b$, and $\mathrm{Cr}$ must be clamped to 255 when they are maximum value.

RGB to $\mathrm{YCbCr}$ Conversion

$\mathrm{YCbCr}$ (256 levels) can be computed directly from 8-bit RGB as follows:

$$
\begin{aligned}
& Y=0.299 R+0.587 G+0.114 B \\
& C b=-0.1687 R-0.3313 G+0.5 B+128 \\
& C r=0.5 R-0.4187 G-0.0813 B+128
\end{aligned}
$$

NOTE - Not all image file formats store image samples in the order $R_{0}, G_{0}, B_{0}$, $\ldots R_{n}, G_{n}, B_{n}$. Be sure to verify the sample order before converting an RGB file to JFIF

\section{YCbCr to RGB Conversion}

RGB can be computed directly from $\mathrm{YCbCr}$ (256 levels) as follows:

$$
\begin{aligned}
& R=Y+1.402(\mathrm{Cr}-128) \\
& G=Y-0.34414(\mathrm{Cb}-128)-0.71414(\mathrm{Cr}-128) \\
& B=Y+1.772(\mathrm{Cb}-128)
\end{aligned}
$$

\section{Image Orientation}

In JFIF files, the image orientation is always top-down. This means that the first image samples encoded in a JFIF file are located in the upper left hand corner of the image and encoding proceeds from left to right and top to bottom. Top-down orientation is used for both the full resolution image and the thumbnail image.

The process of converting an image file having bottom-up orientation to JFIF must include inverting the order of all image lines before JPEG encoding.

\section{Spatial Relationship of Components}

Specification of the spatial positioning of pixel samples within components relative to the samples of other components is necessary for proper image post processing and accurate image presentation. In JFIF files, the position of the pixels in subsampled components are defined with respect to the highest resolution component. Since components must be sampled orthogonally (along rows and columns), the spatial position of the samples in a given subsampled component may be determined by specifying the horizontal and vertical offsets of the first sample, i.e. the sample in the upper left corner, with respect to the highest resolution component.

The horizontal and vertical offsets of the first sample in a subsampled component, Xoffset $_{\mathrm{i}}$ $[0,0]$ and Yoffset $_{i}[0,0]$, are defined to be:

$$
\begin{aligned}
& \text { Xoffset }_{i}[0,0]=\left(\left(\text { Nsamples }_{\text {ref }} / \text { Nsamples }_{\mathrm{j}}\right) / 2\right)-0.5 \\
& \text { Yoffset }_{i}[0,0]=\left(\left(\text { Nlines }_{\text {ref }} / \text { Nlines }_{i}\right) / 2\right)-0.5
\end{aligned}
$$




\section{where}

Nsamples ${ }_{\text {ref }}$ is the number of samples per line in the largest component; Nsamples $_{\mathrm{i}}$ is the number of samples per line in the ith component; Nlines $_{\text {ref }}$ is the number of lines in the largest component; Nlines ${ }_{i}$ is the number of lines in the ith component.

Proper subsampling of components incorporates an anti-aliasing filter which reduces the spectral bandwidth of the full resolution components. Subsampling can easily be accomplished using a symmetrical digital filter with an even number of taps (coefficients). A commonly used filter for $2: 1$ subsampling utilizes two taps $(1 / 2,1 / 2)$.

As an example, consider a 3 component image which is comprised of components having the following dimensions:

Component 1: 256 samples, 288 lines

Component 2: 128 samples, 144 lines

Component 3: 64 samples, 96 lines

In a JFIF file, centers of the samples are positioned as illustrated below:

$\mathbf{X}$

$\mathbf{x}$

$\mathbf{x}$

$\mathbf{X}$

$\times \quad 0 \quad x$

$\mathbf{X}$

X

component 1

component 2

X

X

$x$

$\mathrm{x}$

O

Component 3

x

x

x

$\mathbf{x}$

NOTE - This definition is compatible with industry standards such as Postscript Level 2 and QuickTime. This definition is not compatible with the conventions used by CCIR Recommendation 601-I and other digital video formats. For these formats, pre-processing of the chrominance components is necessary prior to compression in order to ensure accurate reconstruction of the compressed image.

\section{JPEG File Interchange Format Specification}

The syntax of a JFIF file conforms to the syntax for interchange format defined in Annex $B$ of ISO DIS 10918-1. In addition, a JFIF file uses APPO marker segments and constrains certain parameters in the frame header as defined below. 
X'FF', sol

X'FF', APPO, length, identifier, version, units, Xdensity, Ydensity, Xthumbnail, Ythumbnail, (RGB) n

\begin{tabular}{|c|c|c|}
\hline length & ( 2 bytes) & $\begin{array}{l}\text { Total APPO field byte count, including the byte } \\
\text { count value ( } 2 \text { bytes), but excluding the APPO } \\
\text { marker itself }\end{array}$ \\
\hline identifier & (5 bytes) & $\begin{array}{l}=X^{\prime} 4 A^{\prime}, X^{\prime} 46^{\prime}, X^{\prime} 49^{\prime}, X^{\prime} 466^{\prime}, X^{\prime} 00^{\prime} \\
\text { This zero terminated string ("JFIF") uniquely } \\
\text { identifies this APPO marker. This string shall } \\
\text { have zero parity (bit } 7=0 \text { ). }\end{array}$ \\
\hline version & ( 2 bytes) & $\begin{array}{l}=X^{\prime} 0102^{\prime} \\
\text { The most significant byte is used for major } \\
\text { revisions, the least significant byte for minor } \\
\text { revisions. Version } 1.02 \text { is the current released } \\
\text { revision. }\end{array}$ \\
\hline units & (1 byte) & $\begin{array}{l}\text { Units for the } X \text { and } Y \text { densities } \\
\text { units }=0: \text { no units, } X \text { and } Y \text { specify the pixel } \\
\text { units }=1: X \text { and } Y \text { are dots per inch } \\
\text { units }=2: X \text { and } Y \text { are dots per } \mathrm{cm}\end{array}$ \\
\hline Xdensity & ( 2 bytes) & Horizontal pixel density \\
\hline Ydensity & ( 2 bytes) & Vertical pixel density \\
\hline Xthumbnail & (1 byte) & Thumbnail horizontal pixel count \\
\hline Ythumbnail & (1 byte) & Thumbnail vertical pixel count \\
\hline$(R G B)_{n}$ & ( $3 n$ bytes) & $\begin{array}{l}\text { Packed (24-bit) RGB values for the thumbnail } \\
\text { pixels, } n=\text { Xthumbnail * Ythumbnail }\end{array}$ \\
\hline
\end{tabular}

[Optional JFIF extension APPO marker segment(s) - see below]

$\underline{X}$ 'FF', SOF $\underline{n}$ length. frame parameters

Number of components $\quad \mathrm{Nf}=1$ or 3

1st component $\quad C_{1} \quad=1=Y$ component

2nd component $\quad \mathrm{C}_{2} \quad=2=\mathrm{Cb}$ component

3rd component $\quad \mathrm{C}_{3} \quad=3=\mathrm{Cr}$ component

$X$ 'FF', EOI 


\section{JFIF Extension APPO Marker Segment}

Immediately following the JFIF APPO marker segment may be a JFIF extension APPO marker. This JFIF extension APPO marker segment may only be present for JFIF versions 1.02 and above. The syntax of the JFIF extension APPO marker segment is:

$\underline{X}$ 'FF', APPO,. Length, identifier, extension code, extension data

length (2 bytes) Total APPO field byte count, including the byte count value ( 2 bytes), but excluding the APPO marker itself

identifier

(5 bytes)

$=X^{\prime} 4 A^{\prime}, X^{\prime} 46^{1}, X^{\prime} 58^{\prime}, X^{\prime} 58^{\prime}, X^{\prime} 00^{\prime}$

This zero terminated string ("JFXX") uniquely identifies this APPO marker. This string shall have zero parity (bit $7=0$ ).

extension_code (1 byte)

= Code which identifies the extension. In this version, the following extensions are defined:

$=X^{\prime} 10^{\prime}$ Thumbnail coded using JPEG

$=X^{\prime} 11^{\prime}$ 'Thumbnail stored using 1 byte/pixel

$=X^{\prime} 13^{\prime}$ 'Thumbnail stored using 3 bytes/pixel

extension_data (variable) = The specification of the remainder of the JFIF extension APPO marker segment varies with the extension. See below for a specification of extension_data for each extension.

JFIF Extension: Thumbnail coded using JPEG

This extension supports thumbnails compressed using JPEG. The compressed thumbnail immediately follows the extension-code $(X ' 10)$ in the extension_data field and the length of the compressed data must be included in the JFIF extension APPO marker length field.

The syntax of the extension_data field conforms to the syntax for interchange format defined in Annex B of ISO DIS 10918-1. However, no "JFIF" or "JFXX" marker segments shall be present. As in the full resolution image of the JFIF file, the syntax of extension_data constrains parameters in the frame header as defined below:

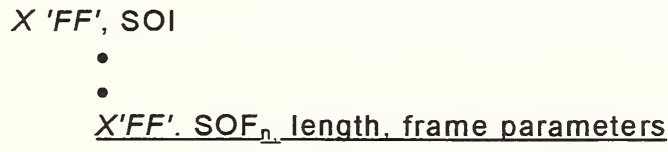

$\begin{array}{ll}\text { Number of components } & \mathrm{Nf}=1 \text { or } 3 \\ \text { 1st component } & \mathrm{C}_{1}=1=\mathrm{Y} \text { component } \\ \text { 2nd component } & \mathrm{C}_{2}=2=\mathrm{Cb} \text { component } \\ \text { 3rd component } & \mathrm{C}_{3}=3=\mathrm{Cr} \text { component } \\ \text { - } & \\ \text { - } & \end{array}$




\section{JFIF Extension: Thumbnail stored using one byte per pixel}

This extension supports thumbnails stored using one byte per pixel and a color palette in the extension_data field. The syntax of extension_data is:

\begin{tabular}{|c|c|c|}
\hline Xthumbnail & (I byte) & Thumbnail horizontal pixel count \\
\hline Ythumbnail & (1 byte) & Thumbnail vertical pixel count \\
\hline palette & (768 bytes) & $\begin{array}{l}\text { 24-bit RGB pixel values for the color palette. } \\
\text { The RGB values define the colors represented } \\
\text { by each value of an } 8 \text {-bit binary encoding }(0- \\
255) \text {. }\end{array}$ \\
\hline$(\text { pixel })_{n}$ & (n bytes) & $\begin{array}{l}\text { 8-bit values for the thumbnail pixels } \\
n=\text { Xthumbnail * Ythumbnall }\end{array}$ \\
\hline
\end{tabular}

\section{JFIF Extension: Thumbnail stored using three bytes per pixel}

This extension supports thumbnails stored using three bytes per pixel in the extension_data field. The syntax of extension_data is:

$\begin{array}{lll}\text { Xthumbnail } & (1 \text { byte }) & \text { Thumbnail horizontal pixel count } \\ \text { Ythumbnail } & (1 \text { byte }) & \text { Thumbnail vertical pixel count } \\ (\mathrm{RGB})_{n} & \text { (3n bytes) } & \begin{array}{l}\text { Packed }(24-\mathrm{bit}) \mathrm{RGB} \text { values for the thumbnail } \\ \text { pixels, } n=\text { Xthumbnail * Ythumbnail }\end{array}\end{array}$

\section{Useful tips}

- you can identify a JFIF file by looking for the following sequence: $X^{\prime} F F^{\prime}, S O I, X^{\prime} F F^{\prime}$, APPO, <2 bytes to be skipped>, "JFIF", $X^{\prime} 00$ '.

- if you use APPO elsewhere, be sure not to have the strings "JFIF" or "JFXX" right after the APPO marker.

- if you do not want to include a thumbnail, just program Xthumbnail $=$ Ythumbnail $=0$.

- be sure to check the version number in the special APPO field. In general, if the major version number of the JFIF file matches that supported by the decoder, the file will be decodable.

- if you only want to specify a pixel aspect ratio, put 0 for the units field in the special APPO field. Xdensity and Ydensity can then be programmed for the desired aspect ratio. Xdensity = 1 , Ydensity $=1$ will program a 1:1 aspect ratio. Xdensity and Ydensity should always be nonzero. 


\section{Annex F}

An Example of the Use of the Standard

This example contains a transaction information record (Type-1), a user-defined descriptive text record (Type-2), a facial image record (Type-10), and a tattoo image record (Type-10). The pose in the facial image was halfway between a full-face and right profile pose. A compression ratio of approximately $20: 1$ and $25: 1$ was used for the facial record and tattoo records respectively.

TYPE-1 RECORD

*LENGTH (LEN)

*VERSION (VER)

*CONTENT (CNT)

"TYPE OF TRANSACTION (TOT)

*DATE (DAT)

PRIORITY (PRY)

*DESTINATION AGENCY IDENTIFIER (DAI)

*ORIGINATING AGENCY IDENTIFIER (ORI)

*TRANSACTION CONTROL NUMBER (TCN)

"NATIVE SCANNING RESOLUTION (NSR)

*TRANSMITTING RESOLUTION

*LENGTH (LEN)

*IMAGE DESIGNATION CHARACTER (IDC)

USER-DEFINED INFORMATION
$1.01: 151 \stackrel{G}{S}$

$1.02: 0201_{\mathrm{S}}^{\mathrm{G}}$

1.03:1 ${ }_{S}^{U} 3{ }_{S}^{R} 02{ }_{S}^{U} 00{ }_{S}^{R} 10{ }_{S}^{U} 01{ }_{S}^{R} 10{ }_{S}^{U} 02{ }_{S}^{G}$

1.04: $X_{X \times}^{G}$

$1.05: 19960229{ }_{S}^{G}$

$1.06: 1 \stackrel{G}{S}$

1.07:DCFBIWA6Z ${ }_{S}^{G}$

1.08: NY0303000SLAS $01000 \stackrel{G}{S}$

$1.09: 234567 \mathrm{AB}_{S}^{G}$

$1.11: 00.00 \stackrel{G}{S}$

$1.12: 00.00{ }_{S}^{F}$

TYPE-2 RECORD

$2.01: 356 \stackrel{G}{s}$

$2.02: 00 \stackrel{G}{S}$

(338 ASCII TEXT CHARACTERS) ${ }_{S}^{F}$

"MANDATORY FIELDS 


\section{TYPE-10 RECORD (FACIAL)}

\section{ST TYPE-10 RECORD --}

*LENGTH (LEN)

*IMAGE DESIGNATION CHARACTER (IDC)

*IMAGE TYPE (IMT)

*SOURCE AGENCY (SRC)

*PHOTO DATE (PHD)

*HORIZONTAL LINE LENGTH (HLL)

*VERTICAL LINE LENGTH (VLL)

*SCALE UNITS (SLC)

*HORIZONTAL PIXEL SCALE (HPS)

*VERTICAL PIXEL SCALE (VPS)

*COMPRESSION ALGORITHM (CGA)

*COLORSPACE (CSP)

SUBJECT POSE (POS)

POSE OFFSET ANGLE (POA)

PHOTO DESCRIPTION (PXS)

*IMAGE DATA (DAT)

SOI \& APPO Marker Segment

Compressed Image Data

End Of Image Marker Code
$10.001: 14601_{S}^{G}$

$10.002: 01_{S}^{G}$

10.003:FACE ${ }_{S}^{G}$

10.004:NY0303000SLAS0 $1000_{S}^{G}$

$10.005: 19960229{ }_{S}^{G}$

$10.006: 480_{S}^{G}$

$10.007: 600 \stackrel{G}{S}$

10.008:0 ${ }_{S}^{G}$

$10.009: 1 \stackrel{G}{S}$

$10.010: 1 \stackrel{G}{S}$

10.011:JPEGB ${ }_{S}^{G}$

10.012:YCC ${ }_{S}^{G}$

10.020: $\mathrm{A}_{S}^{G}$

$10.021:-45_{S}^{G}$

10.022: HAT ${ }_{S}^{R}$ GLASSES ${ }_{S}^{G}$

10.999:

X'FFD8', X'FFEO' X'0010'

$X^{\prime} 4 A 46494600^{\prime}, X^{\prime} 01022^{\prime}, X^{\prime} 00^{\prime}, X^{\prime} 0001^{\prime}, X^{\prime} 00011^{\prime}$

$X^{\prime} 00^{\prime}, X^{\prime} 00^{\prime}$,

(FACIAL DATA COMPRESSED @ 20:1 TO 14382 BYTES)

$\mathrm{X}^{\prime}$ FFD ${ }^{\prime}{ }_{S}$ 


\section{TYPE-10 RECORD (TATTOO)}

\section{ND TYPE-10 RECORD --}

*LENGTH (LEN)

*IMAGE DESIGNATION CHARACTER (IDC)

*IMAGE TYPE (IMT)

*SOURCE AGENCY (SRC)

*PHOTO DATE (PHD)

*HORIZONTAL LINE LENGTH (HLL)

*VERTICAL LINE LENGTH (VLL)

*SCALE UNITS (SLC)

*HORIZONTAL PIXEL SCALE (HPS)

*VERTICAL PIXEL SCALE (VPS)

*COMPRESSION ALGORITHM (CGA)

*COLORSPACE (CSP)

*NCIC DESIGNATION CODE (SMT)

SCAR/MARK/TATTOO SIZE (SMS)

TATTOO DESCRIPTION (TAT)

COLORS PRESENT (COL)

*IMAGE DATA (DAT)

SOI \& APPO Marker Segment

Compressed Image Data

End Of Image Marker Code
$10.001: 11797_{S}^{G}$

$10.002: 02_{S}^{G}$

10.003:TATTOO ${ }_{S}^{G}$

10.004: NY0303000SLAS $01000_{S}^{G}$

$10.005: 19960229_{S}^{G}$

$10.006: 480_{S}^{G}$

$10.007: 600 \stackrel{G}{S}$

$10.008: 0_{S}^{G}$

10.009:1 ${ }_{S}^{G}$

$10.010: 1^{G}$

10.011:JPEGB ${ }_{S}^{G}$

10.012:YCC ${ }_{S}^{G}$

10.040:TAT R ANKL $\stackrel{G}{S}$

$10.041: 5_{S}^{U} 10 \stackrel{G}{S}$

10.042: TATTOO ${ }_{S}^{U}$ OBJECT ${ }_{S}^{U}$ NATURE $_{S}^{U}$ SUNBURST $_{S}^{R}$

TATTOO ${ }_{S}^{U}$ HUMAN ${ }_{S}^{U}$ ROLES ${ }_{S}^{U}$ KNIGHT $_{S}^{G}$

10.043: MULTI $_{s}^{R}$ GRAY ${ }_{s}^{U}$ BLUE $_{s}^{G}$

10.999:
X'FFD8', X'FFEO' X'0010'

$X^{\prime} 4 A 46494600^{\prime}, X^{\prime} 01022^{\prime}, X^{\prime} 00^{\prime}, X^{\prime} 0001^{\prime}, X^{\prime} 0001^{\prime}$, $X^{\prime} 00^{\prime}, X^{\prime} 00^{\prime}$,

(TATOO DATA COMPRESSED @ 25:1 TO 11499 BYTES)

X'FFD9' $_{S}^{F}$ 

